\title{
Counseling a Patient with the Antenatal Diagnosis of a Cerebellar Abnormality and a Pharyngeal Cyst
}

\author{
Lissa Francois, MD ${ }^{1}$ Rachanna Tyagi, MD ${ }^{2}$ Thomas Hegyi, MD 3 Joaquin Santolaya-Forgas, MD, $\mathrm{PhD}^{1}$ \\ 1 Department of Obstetrics, Gynecology and Reproductive Sciences, \\ Rutgers-Robert Wood Johnson Medical School, New Brunswick, New \\ Jersey \\ 2 Department of Pediatric Neurosurgery, Rutgers-Robert Wood

\begin{abstract}
Address for correspondence Professor Joaquin Santolaya-Forgas, MD, $\mathrm{PhD}$, Chair of the Center for OBGYN Research and Mentorship, RutgersRobert Wood Johnson Medical School, 125 Paterson Street, Room
\end{abstract} \\ 2151, New Brunswick, NJ 08901 (e-mail: santoljo@rwjms.rutgers.edu).
} Johnson Medical School, New Brunswick, New Jersey

3 Division of Neonatology, Department of Pediatrics, SIDS Center of New Jersey, Rutgers-Robert Wood Johnson Medical School, New Brunswick, New Jersey

Am J Perinatol Rep 2014;4:e89-e92.

\begin{abstract}
Keywords

- cerebellar anomaly

- clivus bone

- encephalocele

- prenatal counseling

- posterior fossa abnormality

Introduction Prenatal counseling with regards to the prognosis of a cerebellar abnormality is hindered not only by the diverse clinical presentations but also by the presence of subtle findings. We present a case of a distinct combination of asymmetric cerebellar hypoplasia secondary to an anterior meningoencephalocele through a clival defect that caused a severe airway obstruction in the newborn.

Case Description A 21-year-old gravida 4 para 0 mother with a dichorionic-diamniotic twin pregnancy was referred for a second trimester sonographic survey. An asymmetric cerebellar hypoplasia, mega cisterna magna, and a pharyngeal cystic mass were noted on twin A. Magnetic resonance imaging report confirmed posterior fossa abnormalities and shed no light on the differential diagnosis of the cystic mass. The pregnancy ended by Cesarean delivery at 32 weeks' gestation after a preterm premature rupture of the membranes. Twin A had a severe airway obstruction. Postnatal evaluation confirmed a midline anterior meningoencephalocele through a defect in the clivus. The microarray chromosomal analysis demonstrated a $5 q 15$ variant with uncertain clinical significance. Conclusion Antenatal recognition of the unique combination of a cerebellar hypoplasia with a pharyngeal cyst can impact the prenatal counseling as well as neonatal management.
\end{abstract}

\section{Case Description}

We describe a case of a newborn with airway obstruction due to a midline meningoencephalocele secondary to a defect in the clivus. The mother is a 21 -year-old gravida 4 para 0 that presented at 23 weeks' gestation for a sonographic survey. A spontaneous dichorionic-diamniotic twin pregnancy was diagnosed. Both the fetuses were females, concordant in size and with appropriate standard biometry for gestational age. Fetus A had asymmetric cerebellar hypoplasia with the transcerebellar diameter measuring $18 \mathrm{~mm}$. The cisterna magna was prominent measuring $12 \mathrm{~mm}$ in anterior-posterior diameter. In addition, a $12 \mathrm{~mm} \times 16 \mathrm{~mm}$ cystic mass was visualized within the fetal pharynx and at the base of the skull. No anomalies were noted on twin B. Magnetic resonance imaging (MRI) findings confirmed asymmetric hypoplastic cerebellum on twin A with a normal vermis and the mega cisterna magna. No supratentorial abnormalities or received

May 19, 2014

accepted after revision

August 18, 2014

published online

November 18, 2014
DOI http://dx.doi.org/

10.1055/s-0034-1394151. ISSN 2157-6998.
Copyright $\odot 2014$ by Thieme Medical Publishers, Inc., 333 Seventh Avenue, New York, NY 10001, USA. Tel: +1(212) 584-4662.
License terms

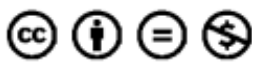


evidence of dysraphism was reported. No comment was made regarding the cystic mass anterior to the spine and within the fetal pharynx. The patient was informed that asymmetric cerebellar hypoplasia is a rare clinical entity with etiologies attributed to focal insults such as infarct, hemorrhage, or infection. Prenatal counseling regarding prognosis is based on the percentage of cerebellar surface loss and vermis involvement. The patient declined prenatal genetic analysis and opted to continue the pregnancy. On follow-up ultrasound the asymmetric cerebellar hypoplasia and pharyngeal cyst were confirmed together with polyhydramnios. The patient was admitted to the hospital at 29 weeks' gestation with preterm premature rupture of membrane on twin A. At 32 weeks' gestation an uncomplicated Cesarean delivery was performed after spontaneous onset of labor with twin $A$ in breech presentation. Immediately after birth, twin A developed intermittent episodes of desaturation. During the intubation by the neonatologist a mass was visualized in the upper airway. In the neonatal intensive care unit, she required ventilator assistance and because of some abnormal movements of the extremities a neurological evaluation was requested. No epileptiform activity was noted on the electroencephalogram and the supratentorial activity was normal for developmental age. Because of the continued respiratory difficulties a pediatric otolaryngologist performed an endoscopic evaluation and noted a retropharyngeal mass obstructing the airway. MRI demonstrated a cerebellar/brainstem malformation (-Fig. 1b) and the computed tomography (CT) scan of the neck demonstrated a bony defect in the midline anterior to the cerebellum that allowed for protrusion of soft tissue into the pharynx. Thus, the airway obstruction was caused by an anterior meningoencephalocele that contained a small component of the pons extending through a clival defect. A CT myelogram was performed and dye was visualized within the pharyngeal cyst with no evidence of an active cerebrospinal fluid leak. A closer inspection of the antenatal MRI pictures demonstrated that the lesion was already present at 23 weeks' gestation (- Fig. 1a). No other congenital anomalies were found on physical evaluation or on thoracic or abdominal complementary imaging. The patient continues to require respiratory support due to the inability for an adequate oral exposure for a primary repair of the meningoencephalocele. A simple tracheostomy was performed with the plan for definitive repair and reconstruction of the clivus when the infant is large enough to tolerate the procedure. An interstitial deletion spanning $803 \mathrm{~kb}$, mapping at the long arm of chromosome 5 (5q15) involving the RIKO2 gene, was reported after chromosomal microarray analysis. Twin B had an uncomplicated neonatal course.

\section{Discussion}

Antenatal sonographic evaluations can be used to detect cerebellar abnormalities. The most common abnormalities are either the abnormal shape of the cerebellum or cerebellar and/or cisterna magna measurements found to be out of the normal range for gestational age. It is our opinion that this premature newborn survived owing to the advances in neonatal care and the modern multidisciplinary approach in perinatal medicine. The mother has voiced understanding of the very limited clinical information concerning the longterm effect of such an anomaly on central nervous system development. She is also aware of the uncertainty of the longterm neurologic outcome with structural abnormalities involving the pons and the cerebellum. The mother would like to know the reason for this anomaly and a clinical strategy for a future pregnancy.

We propose the following sequence to explain a posterior fossa anomaly with a meningoencephalocele due to a defect of the clivus. We begin by mentioning that posterior fossa anomalies occur in about 1 in 10,000 pregnancies with a spectrum of clinical entities ranging from normal variants to more severe anomalies. ${ }^{1}$ The distinct combination of asymmetric cerebellar hypoplasia with a clival defect and anterior meningoencephalocele is very rare. We can then review the postnatal MRI and CT myelogram images that demonstrate the anomalous and direct communication of the exophytic meningoencephalocele with the posterior fossa and discussed that it is plausible that this field defect originated from a primary maldevelopment with the rhombomere

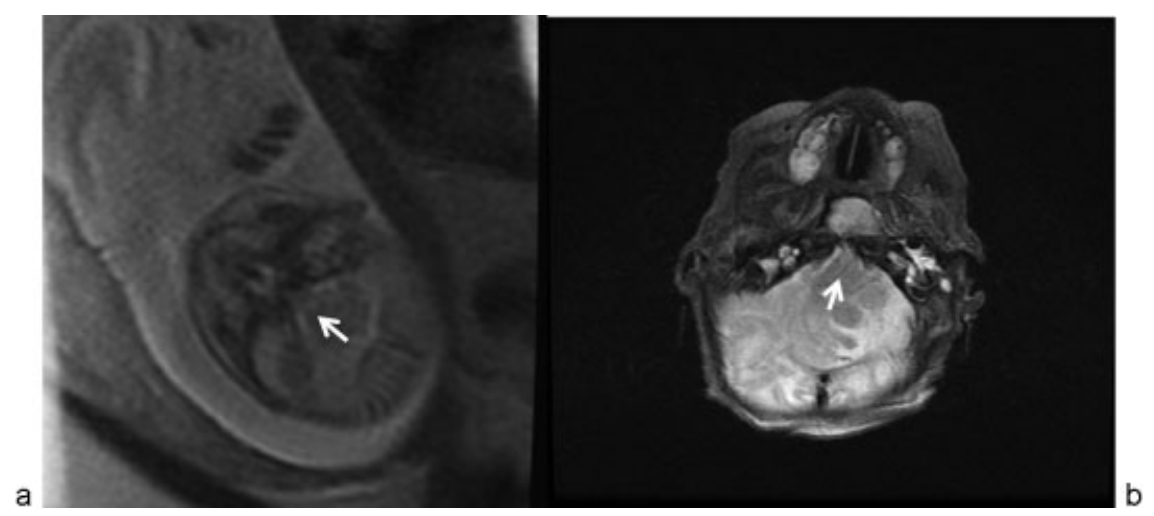

Fig. 1 The exophytic anterior meningoencephalocele is demonstrated in these prenatal (a) and postnatal (b) MRI pictures. The site of the defect in the clivus bone that allows for the direct communication of the pharyngeal mass and the brain is highlighted with arrows. MRI, magnetic resonance imaging. 
disrupting the normal configuration of the notochord. The involvement of the clivus, together with the exophytic anterior meningocele-containing mesenchymal and neural tissues suggests that the lesion had a very early onset. Because there are no dorsal cutaneous stigmata of a tethered cord and the posterior skull/spine are intact, we disregard the possibility of a primary defect in neurulation. ${ }^{2,3}$ We can also rule out a Thornwaldt cyst because there is no abnormal adhesion of the notochord remnants to the pharyngeal endoderm lining and the cyst is not filled with mucous secretion. ${ }^{4}$ Finally, the remnant abnormality known as the "split cord malformation" was ruled out because a midline cleft persists through neurulation in this condition leading to a basicranial diplomyelia with a fibrous stalk splitting the brainstem. Rather, in this case the intrathecal contents are displaced ventrally instead of having anomalous mesenchymal tissues extending dorsally within the neural space. ${ }^{5}$

Experimental data obtained from model organisms for neural developmental investigations can be used to discuss recurrence risk and a future clinical strategy. Indeed, the primary formation of the cerebellum, brain, and neural tube depends on a sequence of events determined by: (1) proper planar cell polarity, ${ }^{2}(2)$ correct midline differentiation of the notochord and the floor plate of the primary neural tube, ${ }^{6}(3)$ genetic polymorphisms that increase the susceptibility to environmental factors capable of disrupting neural tube closure points, ${ }^{7}$ (4) excessive and inadequate induction of apoptotic cell death modifying the morphogenetic processes, ${ }^{8}(5)$ epigenetic effects, for example, folate replacement has been shown to reduce the effect of methylation inhibition on mesenchymal density with exencephaly and other neural tube defects. ${ }^{9}$ In this patient, an interstitial deletion in chromosome 5 (5q15) was diagnosed using chromosomal microarray analysis. Metaphase fluorescence in situ hybridization studies of the deleted interval (RP11194D10) confirmed the deletion that includes the RIOK2 gene. Thus, this genetic abnormality could reduce by half the function of the RIOK2 gene with a relative loss of function of a serine protein kinase that is the transcript of this gene with undetermined target proteins. However, the correlation between heterozygous deletions of this genomic region that contains the RIOK2 gene with anomalous ossification of the clivus and direct exophytic meningoencephalocele has not been reported to our knowledge. Therefore, the causal relationship between the genotype and the phenotype cannot be established at this time. Moreover, a paternal analysis was not possible and inheritance of the $5 q 15$ microdeletion from a normal parent could not be ruled out.

Regarding a strategy for future pregnancies, we recommended preconception folate supplementation and an early prenatal sonographic screening with the option of prenatal genetic diagnosis during the first trimester of pregnancy.

In summary, cerebellar malformations should be classified based on the anatomical appearance and associated findings. We present a case with asymmetric cerebellar hypoplasia, mega cisterna magna and a cyst within the fetal pharynx noted during the 23 week sonographic survey. The pharyngeal cyst was an anterior meningoencephalocele and lead to upper respiratory tract obstruction in the newborn with eventual tracheostomy. The pharyngeal obstruction most likely caused polyhydramnios, premature rupture of the membranes, and a premature delivery. The infant has an interstitial deletion in the long arm of chromosome 5 after microarray analysis. Presently, this genetic finding is considered as a variant with unknown clinical significance.

\section{References}

1 Long A, Moran P, Robson S. Outcome of fetal cerebral posterior fossa anomalies. Prenat Diagn 2006;26(8):707-710

2 Copp AJ. Neurulation in the cranial region-normal and abnormal. J Anat 2005;207(5):623-635

3 Greene ND, Copp AJ. Development of the vertebrate central nervous system: formation of the neural tube. Prenat Diagn 2009;29(4):303-311

4 Baisakhiya N, Deshmukh P, Pawar V. Tornwaldt cyst: a cause of neck pain and stiffness. Indian J Otolaryngol Head Neck Surg 2011; 63(Suppl 1):147-148

5 Rustamzadeh E, Graupman PC, Lam CH. Basicranial diplomyelia: an extension of the split cord malformation theory. Case report. J Neurosurg 2006;104(5, Suppl)362-365

6 Carroll EA, Gerrelli D, Gasca S, et al. Cordon-bleu is a conserved gene involved in neural tube formation. Dev Biol 2003;262(1): 16-31

7 Fleming A, Copp AJ. A genetic risk factor for mouse neural tube defects: defining the embryonic basis. Hum Mol Genet 2000;9(4): 575-581

8 Massa V, Savery D, Ybot-Gonzalez P, et al. Apoptosis is not required for mammalian neural tube closure. Proc Natl Acad Sci U S A 2009; 106(20):8233-8238

9 Dunlevy LP, Burren KA, Mills K, Chitty LS, Copp AJ, Greene ND. Integrity of the methylation cycle is essential for mammalian neural tube closure. Birth Defects Res A Clin Mol Teratol 2006; 76(7):544-552 